otras situaciones (2-4), con frecuencia entre el 0,5 y 2\%, y aparición a las 24-72 horas del accidente o intervención. Cursa clásicamente con compromiso de la función respiratoria, aparición de petequias en distintas localizaciones, alteraciones del nivel de conciencia y en ocasiones trombocitopenia $(1,5)$. No existe ninguna prueba de laboratorio específica que lo caracterice, siendo por tanto clave el diagnóstico clínico, debiendo ser sospechado tras descartar los posibles motivos que pueden ocasionar una alteración en el nivel de conciencia en el contexto de un paciente politraumatizado $(1,5,6)$.

Las alteraciones respiratorias son las más frecuentes en el SEG, apareciendo casi en el $100 \%$, ya que los émbolos grasos afectan inicialmente a los capilares pulmonares, pudiendo posteriormente atravesar la circulación sistémica, alcanzando riñón o cerebro, provocando embolización a dichos niveles $(6,7)$.

Los émbolos en los capilares pulmonares originan una disminución de la presión arterial sistólica e incremento de la presión capilar pulmonar, ambos transitorios, causando un descenso en la proporción entre la presión parcial arterial de oxigeno y la concentración de oxigeno inspirado, con un incremento final del gradiente alveolo arterial (5-8). Las manifestaciones neurológicas son debidas a la migración de los émbolos al cerebro una vez sobrepasada la barrera capilar pulmonar, aunque existen algunos casos de embolismo graso masivo que se asocian a foramen oval permeable ( $27 \%$ de las autopsias en adultos), produciéndose un embolismo paradójico a través de shunt arteriovenoso que origina la embolización cerebral sin afectación inicial de los capilares pulmonares $(8,9)$. En el caso expuesto existe una llamativa discordancia entre la afectación neurológica y la respiratoria, sin haber podido demostrar la presencia de defectos septales cardiacos como explicación al modo de presentación de este síndrome.

En el parénquima cerebral los émbolos grasos provocan por una parte obstrucción de la microcirculación (produciendo cambios isquémicos), y por otra una respuesta inflamatoria desencadenada por mediadores químicos que son liberados tanto desde las plaquetas, como desde las células dañadas por la hipoxia y por la hipoperfusión. Se produce un incremento local de los mediadores que conlleva una disrupción de la membrana basal del capilar y un daño en el tejido subyacente $(1,5-7)$. Su diagnóstico se establece mediante la TAC cerebral que durante la fase aguda muestra punteados hiperdensos difusos en la sustancia blanca, con o sin coexistencia de edema cerebral. Cuando la TAC es normal es recomendable practicar una RM donde se aprecian unas lesiones puntiformes de baja intensidad en T1 e hiperintensas en T2 distribuidas en la sustancia blanca, unión corticomedular, cuerpo calloso y ganglios de la base, que no se refuerzan con el empleo de gadolinio, traduciendo edema vasogénico y citotóxico asociado a la muerte celular durante la fase aguda del embolismo graso $(7,10)$. El seguimiento de esta entidad se realiza mediante RM demostrando la resolución de las lesiones. Diversos estudios sugieren que la RM es el método diagnostico inicial de elección con mayor utilidad y eficiencia para detectar y cuantificar las lesiones en el síndrome de embolia grasa, y para evaluar la severidad del daño cerebral.

El SEG posee habitualmente buen pronóstico, aunque se asocia a mortalidad y a secuelas importantes en algunos casos, debiendo ser diagnosticado precozmente con el fin de prevenir posibles complicaciones, siendo de utilidad la identificación de pacientes con riesgo de presentarlo (5). El tratamiento del mismo requiere medidas de soporte e ingreso en UCI para asegurar una adecuada oxigenación, estabilización hemodinámica y profilaxis de la trombosis venosa profunda. La estabilización intramedular precoz de las fracturas no parece modificar el riesgo de aparición de este síndrome (1). La mortalidad de esta entidad ha disminuido considerablemente desde la aparición de las UCI's, y la realización de un tratamiento (y por tanto de un diagnóstico) precoz conlleva un pronóstico favorable $(1,5)$.
B. Obón Azuara, I. Gutiérrez Cía, P. Luque Gómez, M. A. Navarro Hernández

Servicio de Medicina Intensiva. Hospital Clínico Universitario. Zaragoza

1. Habashi NM, Andrews PL, Scalea TM. Therapeutic aspects of fat embolism syndrome. Injury 2006; 37 (Supl. 4): S68-73.

2. Ajzan A, Modine T, Punjabi P, Ganeshalingam K, Philips G, Gourlay T. Quantification of fat mobilization in patients undergoing coronary artery revascularization using off-pump and on-pump techniques. J Extra Corpor Technol 2006; 38: 116-21.

3. El-Ali KM, Gourlay T. Assessment of the risk of systemic fat mobilization and fat embolism as a consequence of liposuction: ex vivo study. Plast Reconstr Surg 2006; 117: 2269-76.

4. Tolentino LF, Tsai SF, Witt MD, French SW. Fatal fat embolism following amphotericin B lipid complex injection. Exp Mol Pathol 2004; 77: 246-8.

5. Zhou DS, Wang F, Wang BM, Wang LB, Li LX, Xu SH, et al. The diagnosis and treatment of severe cerebral fat embolism. Chin J Traumatol 2003; 6: 375-8.

6. Aydin MD, Akcay F, Aydin N, Gundogdu C. Cerebral fat embolism: pulmonary contusion is a more important etiology than long bone fractures. Clin Neuropathol 2005; 24: 86-90.

7. Sasano N, Ishida S, Tetsu S, Takasu H, Ishikawa K, Sasano H, Katsuya H. Cerebral fat embolism diagnosed by magnetic resonance imaging at one, eight, and 50 days after hip arthroplasty: a case report. Can J Anaesth 2004; 51: 875-9.

8. Riding G, Daly K, Hutchinson S, Rao S, Lovell M, Mc Collum C. Paradoxical cerebral embolisation. An explanation for fat embolism syndrome. J Bone Joint Surg Br 2004; 86: 95-8.

9. Ruiz-Gimeno JI, Ferre MA, Napal MT, Pelegrin F. Prolonged coma due to fat embolism syndrome after fracture of the femur. Rev Esp Anestesiol Reanim 2006; 53: 187-90.

10. Kim YW, Kim HJ, Cho BM, Moon TY, Eun CK. The study of cerebral hemodynamics in the hyperacute stage of fat embolism induced by triolein emulsion. AJNR Am J Neuroradiol 2006; 27: 398-401.

\section{Enfermedad de Madelung asociada a Raynaud}

\section{Sr. Director:}

La enfermedad de Madelung (EM), también denominada lipomatosis simétrica múltiple o lipomatosis benigna simétrica es un cuadro raro caracterizado por el acúmulo de tejido graso no encapsulado en diversas localizaciones, fundamentalmente en la porción superior del tronco y en los miembros superiores. Predomina en varones de edad media, de origen mediterráneo -se estima que en esta área geográfica puede tener una prevalencia (1) de 1 caso por cada 25.000 personas - se asocia a alcoholismo y es de etiología desconocida. En los últimos años se ha comprobado la relación con la utilización de determinados antivirales inibidores de las proteasas en los pacientes VIH positivos. Hay casos aislados en mujeres, en niños y casos familiares. No se dispone de un tratamiento médico satisfactorio. Ni la abstinencia alcohólica ni la dieta hipolipemiante consiguen resolver el problema. La extirpación completa es difícil debido a la falta de encapsulación y a la gran vascularización de estos depósitos. Se ha documentado la 
asociación a polineuropatía y a otros múltiples problemas médicos, algunos de ellos también relacionados con el alcohol, pero no hemos encontrado ninguna publicación en la que se describa la asociación de esta enfermedad con el Raynaud.

Se trata de un varón de 55 años de edad, fumador, que hace 4 años fue estudiado por una hepatopatía etílica crónica con anemia macrocítica relacionada con el consumo de alcohol. Abstinente desde esa fecha. Previamente, con una evolución aproximada de 15 años fue diagnosticado de EM. Relata múltiples bultomas cervicales anteriores y posteriores, así como en las axilas y en la porción proximal de los miembros superiores (Fig. 1). Por ello, fue sometido en varias ocasiones a intervenciones quirúrgicas bajo anestesia general, tanto en la región cervical posterior como en la anterior. Hace 5 años se realizó una electroneuromiografía que fue normal. Asimismo, las radiografías de los ingresos no evidenciaron ensanchamiento mediastínico. En la última revisión nos comenta que desde hace varios años, sin que sepa precisar cuantos, sufre cambios de coloración en los dedos de las manos en relación con la exposición al frío, sugiriendo un Raynaud. Hasta el momento, no sufrió molestias compresivas cervicales. Tiene un IMC de 26, su presión arterial es 130/70 y los parámetros del último control analítico fueron normales.

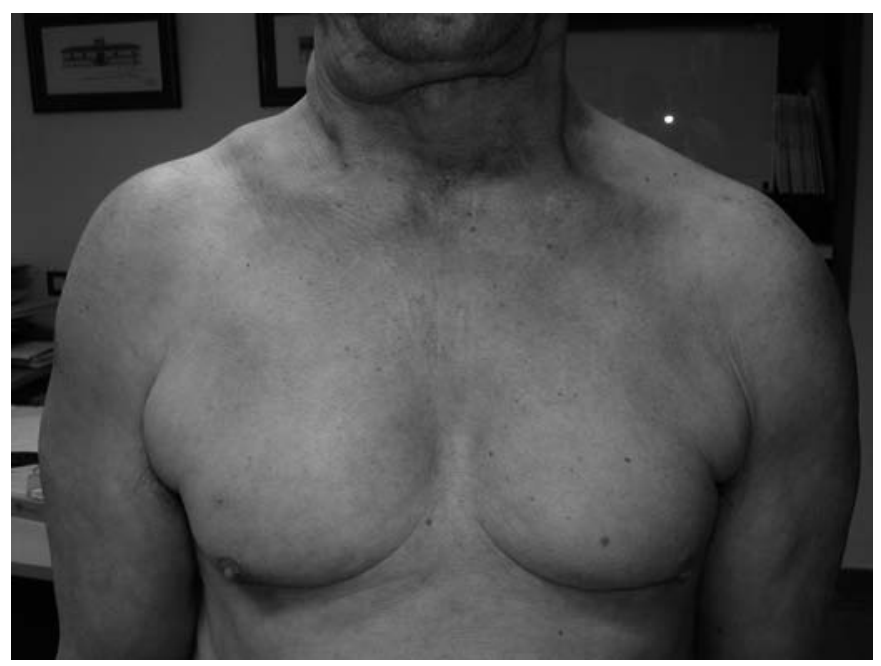

Fig. 1. Depósitos grasos en el cuello, axilas y brazos.

La asociación mejor documentada de la EM es, sin duda alguna, con la polineuropatía, ya sea sensitiva, motora o autonómica que, en estadios avanzados, se puede acompañar de artropatía de Charcot o úlceras neuropáticas (1). Otros problemas neurológicos también descritos en niños y adolescentes son la atrofia cerebelosa (2), el retraso mental (3) y el mielomeningocele (4). Lógicamente, a menudo pueden presentarse otras complicaciones del etilismo como la hepatopatía o la anemia macrocítica (1). Con frecuencia aparecen problemas ligados al síndrome metabólico como pueden ser la diabetes, la hiperuricemia, la hiperlipidemia y la hipertensión arterial. Se han referido, además, otras disfun- ciones endocrinas como son las tiroideas, hipofisarias, adrenales y testiculares. También están documentadas asociaciones con determinados problemas cutáneos como la psoriasis (5), el penfigoide bulloso (6) y el rinofima (7).

Anecdóticamente, está descrita la asociación con apnea del sueño (8) y con el síndrome nefrótico provocado por una glomeruloneritis membranosa (9). Por último, existe algún caso de EM acompañado de claudicación intermitente y úlceras isquémicas en un miembro inferior que, tras una respuesta pobre a las infusiones IV de alprostadil, evolucionó de forma satisfactoria con la colocación de un bypass aortobifemoral (10). Sin embargo, no hemos sido capaces de encontrar ninguna reseña bibliográfica en que aparezca esta enfermedad junto con el Raynaud. En efecto, en una búsqueda sistemática en Pubmed con los parámetros: "benign symmetric lipomatosis" o "Madelung syndrome" o "Madelung disease" o "multiple symmetric lipomatosis" y Raynaud, no apareció ningún item. La enfermedad de Raynaud es una isquemia vascular periférica que aparece como consecuencia de espasmos vasculares desencadenados por el frío, el tabaco y/o el estrés. La clínica se presenta como palidez o cianosis en los dedos. Cuando es secundaria a una enfermedad conocida se conoce como fenómeno de Raynaud. En el caso que nos ocupa ¿se trata de "enfermedad" o de "fenómeno"?

\section{R. Bugarín González, M. Iglesias Caamaño ${ }^{1}$, P. del Río Martín', R. García Rodríguez ${ }^{1}$ I. Vidal Castiñeira ${ }^{1}$, J. M. Pérez Crespo}

Centro de Salud de Calo-Teo. ${ }^{1}$ Unidad Docente de Medicina Familiar y Comunitaria de Santiago de Compostela. A Coruña.

1. Biasi D, Caramaschi P, Carletto A, Baracchino F, Botto M, Pacor ML, et al. Symmetric multiple lipomatosis with Charcot's joint and neuropathic ulcer. Description of a clinical case. Minerva Med 1993; 84: 1359.

2. Castro-Gago M, Alonso A, Pintos-Martínez E, Novo-Rodríguez MI, Blanco-Barca MO, Campos Y, et al. Lipomatosis múltiple simétrica asociada a polineuropatía, atrofia del cerebelo y citopatía mitocondrial. Rev Neurol 2003; 15; 36: 1026-9.

3. Kratz C, Lenard HG, Ruzicka T, Gartner J. Multiple symmetric lipomatosis: An unusual cause of childhood obesity and mental retardation. Eur J Paediatr Neurol 2000; 4: 63-7.

4. Nounla J, Rolle U, Grafe G, Kraling K. Benign symmetric lipomatosis with myelomeningocele in an adolescent: An uncommon associationcase report. J Pediatr Surg 2001; 36: 13.

5. Harkaway KS. Benign symmetric lipomatosis in a patient with severe scalp psoriasis. Cutis 1992; 49: 330-2.

6. Ho MH, Lo KK. Benign symmetric lipomatosis in a Chinese man with bullous pemphigoid. Int J Dermatol 1999; 38: 131-3.

7. Izu R, Gardeazabal J, Bejar J, Aguirre A, Díaz-Pérez JL. A case of the elephant man phenotype with giant rhinophyma and benign symmetric lipomatosis. Clin Exp Dermatol 1994; 19: 531-3.

8. Megevand C, Savoy J, Boudrama A, Meier D. Madelung disease and obstructive sleep apnea syndrome: Apropos of a case. Schweiz Med Wochenschr Suppl 2000; 116: 123-6.

9. Matamala Gimeno C, Perez Calvo JI, Rodriguez Benavente AM, Moros M, Pérez Villaroya JC, Bueno Gómez J. Síndrome nefrótico asociado con lipomatosis simétrica múltiple (Enfermedad de Madelung). An Med Interna (Madrid) 1995; 12: 127-9.

10. Nowakowski T, Bartlewicz J, Nizankowski R, Szczeklik A. Ischemic ulcer in Madelung disease. Pol Arch Med Wewn 2000; 103: 201-4. 